\title{
Hardness, elastic modulus, and structure of very hard carbon films produced by cathodic-arc deposition with substrate pulse biasing
}

\author{
George M. Pharr, Daniel L. Callahan, Shaun D. McAdams, and Ting Y. Tsui \\ Department of Materials Science, Rice University, Houston, Texas 77251-1892 \\ Simone Anders, ${ }^{\text {a) }}$ André Anders, Joel W. Ager III, and lan G. Brown \\ Lawrence Berkeley Laboratory, Berkeley, California 94720 \\ C. Singh Bhatia \\ IBM Corporation, San Jose, California 95193 \\ S. R. P. Silva and John Robertson \\ Department of Engineering, University of Cambridge, Cambridge CB2 1PZ, United Kingdom
}

(received 23 March 1995; accepted for publication 16 November 1995)

\begin{abstract}
The hardness, elastic modulus, and structure of several amorphous carbon films on silicon prepared by cathodic-arc deposition with substrate pulse biasing have been examined using nanoindentation, energy loss spectroscopy (EELS), and cross-sectional transmission electron microscopy. EELS analysis shows that the highest $s p^{3}$ contents $(85 \%)$ and densities $\left(3.00 \mathrm{~g} / \mathrm{cm}^{3}\right)$ are achieved at incident ion energies of around $120 \mathrm{eV}$. The hardness and elastic modulus of the films with the highest $s p^{3}$ contents are at least 59 and $400 \mathrm{GPa}$, respectively. These values are conservative lower estimates due to substrate influences on the nanoindentation measurements. The films are predominantly amorphous with a $\sim 20 \mathrm{~nm}$ surface layer which is structurally different and softer than the bulk. (c) 1996 American Institute of Physics. [S0003-6951(96)01105-6]
\end{abstract}

A cathodic-arc plasma source equipped with a magnetic macroparticle filter is an efficient tool for depositing highquality thin films of metals, alloys, and compounds. To date, the technique has been employed primarily in laboratory settings, but recent advances have led to large units capable of full-scale commercial production. ${ }^{1}$ One material which has received a great deal of attention in cathodic-arc processing is amorphous carbon $(a-C)$. In addition to being chemically inert, electrically insulating, transparent, and having a low coefficient of friction, cathodic-arc carbon is of interest because of its potential as thin coating material with very high hardness. Amorphous carbon has been deposited from a cathodic arc by various groups. ${ }^{2-8}$ Hardness values ranging from $26 \mathrm{GPa}$ to over $60 \mathrm{GPa}$ have been measured by nanoindentation methods. The difficulties of deriving hardness values for such hard, thin films on softer substrates are well recognized. ${ }^{9}$ Nevertheless, the high values reported suggest that $a$-C with very high hardness can be achieved by cathodic-arc deposition.

The key to producing high hardness in amorphous carbon films appears to be in promoting high $s p^{3}$ bond content through careful control of the energy of incident ions during deposition. ${ }^{6,10-14}$ For cathodic-arc deposition, one way to achieve this is by substrate pulse biasing. ${ }^{15}$ Pulse biasing has an important advantage compared to dc bias in that, whereas with dc bias electrical breakdown can result with pulsed biasing the applied voltage can be arbitrarily high (the voltage is switched off before the high voltage plasma sheath expands to dangerous distances).

We have investigated the properties of cathodic-arc carbon as a function of biasing conditions and have found a set

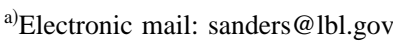

of conditions which produces very hard films. Here, we report the hardness and elastic modulus of these films measured by nanoindentation methods and the structure of the films determined by transmission electron microscopy (TEM) and electron energy loss spectroscopy (EELS).

The carbon films were deposited on silicon substrates using a cathodic-arc plasma source combined with a $90^{\circ}$ bent magnetic macroparticle filter. The source and filter are described in detail elsewhere. ${ }^{16}$ The plasma source was operated in a repetitively pulsed mode with a pulse duration of 5 $\mathrm{ms}$ and a pulse repetition rate of $2 \mathrm{~Hz}$. A cathodic-arc discharge at a current of $300 \mathrm{~A}$ was used to create a fully ionized carbon plasma which streamed out of the source at a velocity of about $2 \times 10^{6} \mathrm{~cm} / \mathrm{s}$, corresponding to an ion energy of about $20 \mathrm{eV}$. The substrates were immersed in this high-density carbon plasma (a density of about $10^{13} \mathrm{~cm}^{-3}$ ) and repetitively pulse biased during the longer plasma pulse at a negative voltage and pulse duration of $2 \mu \mathrm{s}$. Bias voltages of up to $-2 \mathrm{kV}$ and duty cycles of $25 \%$ were employed (i.e., pulse-off times of $6 \mu \mathrm{s}$ ). The silicon substrates were mounted on a water-cooled sample holder which could be biased at the desired voltage. Some properties of the resulting films, such as mass density, coefficient of friction, and residual stress as a function of pulse bias voltage and duty cycle, have been described elsewhere. ${ }^{17,18}$

EELS was performed to determine the $s p^{3}$ bond contents of films deposited at substrate biases of $0,-100,-500$, and $-2000 \mathrm{~V}$. The films were $200 \mathrm{~nm}$ thick and were prepared for analysis by dissolving the silicon substrate in a solution of $\mathrm{HF}, \mathrm{HNO}_{3}$, and deionized (DI) water. The films were analyzed on a Phillips CM30 TEM operated at $100 \mathrm{kV}$ with the EELS collected on a Gatan 666 parallel spectrometer at a collection angle of $10 \mathrm{mrad}$. The valence plasmon 
TABLE I. EELS results.

\begin{tabular}{cccc}
\hline $\begin{array}{c}\text { Bias voltage } \\
(\mathrm{V})\end{array}$ & $\begin{array}{c}\text { Valence plasmon } \\
(\mathrm{eV})\end{array}$ & $\begin{array}{c}\text { Mass density } \\
\left(\mathrm{g} / \mathrm{cm}^{3}\right)\end{array}$ & $\begin{array}{c}s p^{3} \text { content } \\
(\%)\end{array}$ \\
\hline no bias & 29.8 & 2.91 & 81 \\
-100 & 30.3 & 3.00 & 85 \\
-500 & 25.8 & 2.18 & 47 \\
-2000 & 25.6 & 2.14 & 39 \\
\hline
\end{tabular}

and carbon $K$-edge losses were both collected under the same conditions. The valence plasmon gives the valence electron density and, hence, the mass density. The $K$ edge gives the fraction of $\pi^{*}$ states in the conduction band and, hence, the fraction of $s p^{2}$ sites. The fraction of $\pi^{*}$ states was determined by fitting a Gaussian to the first peak around $285 \mathrm{eV}$, including a small Gaussian around $287 \mathrm{eV}$ and a broad Gaussian to the $290 \mathrm{eV}$ edge. The fraction of $s p^{2}$ sites (with an accuracy of $\pm 5 \%$ ) was found by comparing the ratio of the area of the $285 \mathrm{eV}$ peak to the $290 \mathrm{eV}$ peak to that in polycrystalline graphite, which is $100 \% s p^{2}$, and the densities (with an accuracy of $\pm 0.05 \mathrm{~g} / \mathrm{cm}^{3}$ ) by scaling to the plasmon of graphite. ${ }^{6,19}$

The EELS results are summarized in Table I. The $s p^{3}$ content and mass density are greatest for the film deposited at $-100 \mathrm{~V}$, equivalent to an incident ion energy of $120 \mathrm{eV}$. As reported previously, ${ }^{17,18}$ this bias also produces a minimum for the friction coefficient, a maximum for the mass density, and a maximum for the residual compressive stress and agrees well with the results of Fallon et al. ${ }^{6}$ who found maxima in the intrinsic stress, resistivity, and percentage of $s p^{3}$ bonding for cathodic-arc films deposited at ion energies around $140 \mathrm{eV}$. It is also in reasonable agreement with ion energies used to achieve maximum $s p^{3}$ content using other deposition methods. For example, Weiler et al. have recently used a novel plasma beam source to prepare amorphous hydrogenated-carbon films from acetylene and found discrete maxima in the $s p^{3}$ content $(75 \%)$ and the nanoindentation hardness $(61 \mathrm{GPa})$ at an ion energy of $92 \mathrm{eV}$ per incident carbon atom. ${ }^{11}$

The hardness and elastic modulus of several films produced at a bias of $-100 \mathrm{~V}$ were measured using nanoindentation techniques. A method similar to that outlined by Oliver and Pharr was used to carefully establish the tip geometry of the Berkovich diamond indenter to allow for measurements at depths as small as $20 \mathrm{~nm} .{ }^{20}$ Films of three different thickness, 100, 200, and $320 \mathrm{~nm}$, were tested to examine possible influence of the substrate on the measured properties. The films were produced using a two-step process in which a thin layer, approximately $5 \mathrm{~nm}$, was first deposited at the relatively high substrate bias of $-2000 \mathrm{~V}$ followed by growth of the majority of the film at $-100 \mathrm{~V}$. The broad interfacial layer produced in this way serves to improve film adhesion and reduce stress at the interface.

The results of the nanoindentation measurements are summarized in Figs. 1 and 2, where the hardness, $H$, and Young's modulus, $E$, are plotted as a function of the indentation contact depth. For the sake of comparison, the figures also include the results for a $300 \mathrm{~nm} a-\mathrm{C}: \mathrm{H}$ film prepared by

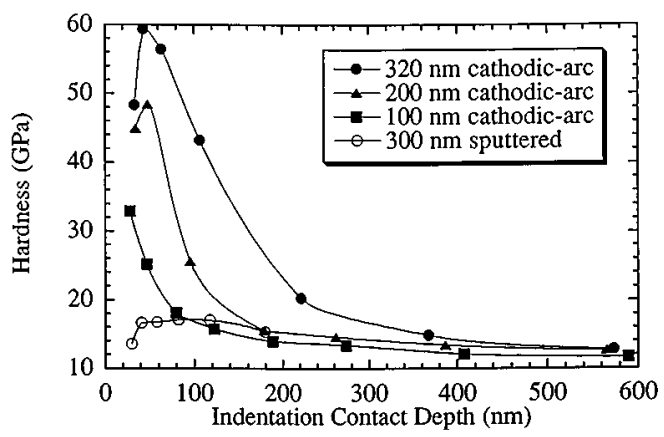

FIG. 1. Nanoindentation measurements of hardness for cathodic-arc and sputter-deposited carbon films on silicon as a function of indentation contact depth.

sputter deposition on silicon in a hydrogen/argon atmosphere. $^{21}$ The $a-\mathrm{C}: \mathrm{H}$ film was $300 \mathrm{~nm}$ thick and contained about 20 at. \% hydrogen, as determined by Rutherford backscattering spectroscopy (RBS) techniques. ${ }^{21}$ The $a-\mathrm{C}: \mathrm{H}$ film is a useful reference, since it was tested at the same time as the cathodic-arc films, was deposited on the same substrate material and has a comparable thickness to the thickest cathodic-arc film.

Examination of Figs. 1 and 2 shows that the hardness and modulus of all four films tend toward those of the silicon substrate at large indenter depths. At small depths, e.g., about $50 \mathrm{~nm}$, properties more representative of the films are obtained. For the sputtered film, the small-depth hardness and elastic modulus are comparable to those of the silicon substrate. In sharp contrast, however, the hardness and modulus of all three cathodic-arc films increase significantly for small depths. The increase is the greatest for the thickest film, whose peak hardness of $59 \mathrm{GPa}$ is approximately four times that of the sputtered film and nearly two thirds of the $90 \mathrm{GPa}$ value commonly quoted for bulk crystalline diamond. ${ }^{22}$ The $59 \mathrm{GPa}$ hardness is approximately a factor of 2 greater than that reported by Falabella et al. ${ }^{5}$ and is similar to the $61 \mathrm{GPa}$ hardness observed by Weiler et al. for hydrogenated carbon films prepared by plasma beam techniques at the optimum ion energy of $92 \mathrm{eV} .^{11}$ The fact that there are no clear plateaus in the small-depth hardness and modulus indicates that the measured values for the cathodic-arc films are not substrate independent. The increase in peak hardness with film

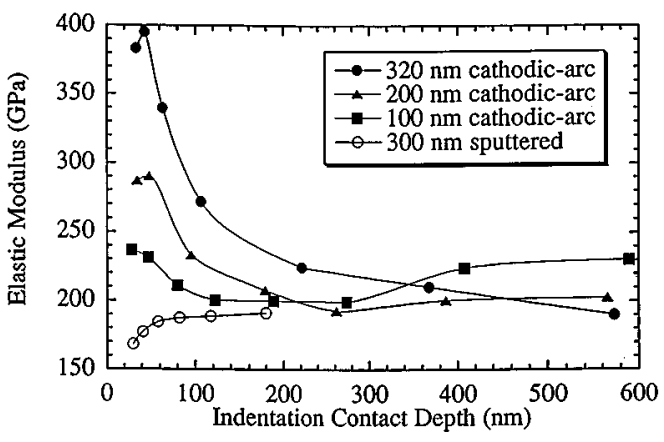

FIG. 2. Nanoindentation measurements of elastic modulus for cathodic-arc and sputter-deposited carbon films on silicon as a function of indentation contact depth. 


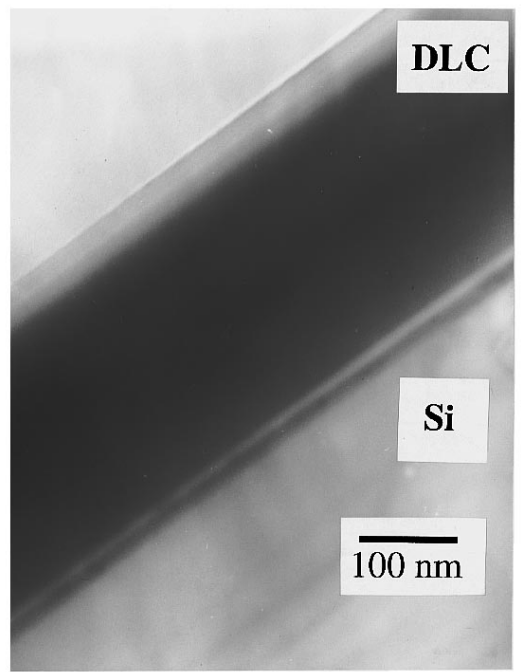

FIG. 3. A bright field TEM image showing a cathodic-arc film in cross section.

thickness is also consistent with this notion. Thus the true film hardness is probably higher than the measurements suggest and may approach that of diamond. Likewise, the elastic modulus of the $320 \mathrm{~nm}$ cathodic-arc film, approximately 400 $\mathrm{GPa}$, also represents a lower limit. For comparison purposes, Young's modulus of polycrystalline diamond is $1140 \mathrm{GPa}^{22}$

The structure of the cathodic-arc films was examined in cross section by transmission electron microscopy. A bright field image of a film approximately $300 \mathrm{~nm}$ thick is given in Fig. 3.

The film has both surface and interface structure. At the surface, a distinctly lighter layer, on the order of $20 \mathrm{~nm}$ thick, is observed in the bright field image. The contrast from this layer is consistent with a lower density material and thus what would probably be a softer form of carbon. A similar, lower density surface layer was observed by Davis et al. ${ }^{23}$ and may arise from the subsurface growth process of films deposited from energetic ions leaving a less dense outer layer. A gradual decrease in brightness is observed at the interface between the surface layer and the bulk of the film. This may arise from a thickness gradient (due to slower bulk milling rates), a density gradient, or a combination of the two effects.

Two distinct layers are observed between the bulk of the film and the silicon substrate, each approximately $10 \mathrm{~nm}$ thick. Immediately adjacent to the silicon substrate is a dark layer corresponding to a $\mathrm{Si}-\mathrm{C}$ reaction layer. This layer is produced by implantation of carbon into the substrate during the $-2000 \mathrm{~V}$ high bias applied during the initial portion of the deposition process. Between the reaction layer and the bulk of the film lies a lighter phase which is consistent with lower density carbon codeposited during the high bias deposition. The interfacial structure is similar to that recently reported by Gerstner et al. ${ }^{8}$

In conclusion, cathodic-arc deposition in conjunction with substrate pulse biasing can be used to produce uniform carbon films with hardnesses of at least $59 \mathrm{GPa}$. The $59 \mathrm{GPa}$ value is a conservative estimate, due to substrate influences on the nanoindentation measurements, and it is conceivable that the hardness approaches that of polycrystalline diamond ( $\sim 90 \mathrm{GPa})$. The substrate bias producing the films with the highest $s p^{3}$ content of $85 \%$ is near $-100 \mathrm{~V}$, consistent with other studies. The films are predominantly amorphous with a thin, soft surface layer approximately $20 \mathrm{~nm}$ thick which is structurally different from the bulk.

This work was supported by the U.S. Department of Energy, Division of Advanced Energy Projects, under Contract No. DE-AC03-76SF00098; by the Advanced Research Projects Agency as part of the National Storage Industry Consortium program in Ultra High Density Recording; and by the Division of Materials Sciences, U. S. Department of Energy, under Contract DE-AC05-84OR21400 with Lockhead Martin Energy Systems, Inc., and through the SHaRE program under Contract DE-AC05-76OR00033 with the Oak Ridge Institute for Science and Education.

${ }^{1}$ D. A. Baldwin and S. Falabella, 38th Society of Vacuum Coaters Technical Conference Proceedings (Society of Vacuum Coaters, Albuquerque, NM, 1995), pp. 309-316.

${ }^{2}$ P. J. Martin, S. W. Filipczuk, R. P. Netterfield, J. S. Field, D. W. Whitnall, and D. R. McKenzie, J. Mater. Sci. Lett. 7, 410 (1988).

${ }^{3}$ D. R. McKenzie, D. Muller, and B. A. Pailthorpe, Phys. Rev. Lett. 67, 773 (1991)

${ }^{4}$ R. Lossy D. L. Pappas, R. A. Roy, J. J. Cuomo, and V. M. Sura, Appl. Phys. Lett. 61, 171 (1992).

${ }^{5}$ S. Falabella, D. B. Boercker, and D. M. Sanders, Thin Solid Films 236, 82 (1993).

${ }^{6}$ P. J. Fallon, V. S. Veerasamy, C. A. Davis, J. Robertson, G. A. J. Amaratunga, W. I. Milne, and J. Koskinen, Phys. Rev. B 48, 4777 (1993).

${ }^{7}$ H. J. Scheibe and B. Schultrich, Thin Solid Films 246, 92 (1994).

${ }^{8}$ E. G. Gerstner, D. P. McKenzie, M. K. Puchert, and P. Y. Trimbell, J. Vac. Sci. Technol. A 13, 406 (1995).

${ }^{9}$ G. M. Pharr and W. C. Oliver, Mater. Res. Soc. Bull. 17, 28 (1992).

${ }^{10}$ J. J. Cuomo, D. L. Pappas, J. Bruley, J. P. Doyle, and K. L. Saenger, J. Appl. Phys. 70, 1706 (1991).

${ }^{11}$ M. Weiler, S. Sattel, K. Jung, H. Ehrhardt, V. S. Veerasamy, and J. Robertson, Appl. Phys. Lett. 64, 2797 (1994).

${ }^{12}$ Y. Lifshitz, S. R. Kasi, and J. W. Rabalais, Phys. Rev. Lett. 62, 1290 (1989).

${ }^{13}$ Y. Lifshitz, G. D. Lempert, and E. Grossman, Phys. Rev. Lett. 72, 2753 (1994).

${ }^{14}$ N. Savvides and T. J. Bell, J. Appl. Phys. 72, 2791 (1992).

${ }^{15}$ A. Anders, S. Anders, I. G. Brown, M. R. Dickinson, and R. A. MacGill, J. Vac. Sci. Technol. B 12, 815 (1994).

${ }^{16}$ A. Anders, S. Anders, and I. G. Brown, Plasma Sources Sci. Technol. 4, 1 (1995).

${ }^{17}$ S. Anders, A. Anders, I. G. Brown, B. Wei, K. Komvopoulos, J. W. AgerIII, and K. M. Yu, Surf. Coat. Technol. 68/69, 388 (1994).

${ }^{18}$ J. W. Ager, S. Anders, A. Anders, and I. G. Brown, Appl. Phys. Lett. 66, 3444 (1995).

${ }^{19}$ S. D. Berger, D. R. McKenzie, and P. J. Martin, Philos. Mag. Lett. 57, 285 (1988).

${ }^{20}$ W. C. Oliver and G. M. Pharr, J. Mater. Res. 7, 1564 (1992).

${ }^{21}$ T. Y. Tsui, G. M. Pharr, W. C. Oliver, Y. W. Chung, E. C. Cutiongco, C. S. Bhatia, R. L. White, R. L. Rhoades, and S. M. Gorbatkin, Mater. Res. Soc. Symp. Proc. 356, 767 (1995).

${ }^{22}$ C. A. Brookes, in Properties of Diamond, edited by J. E. Field (Academic, New York, 383, 1979).

${ }^{23}$ C. A. Davis, G. A. J. Amaratunga, and K. M. Knowles, Surf. Coat. Technol. (to be published). 